

\title{
EL CONOCIMIENTO PEDAGÓGICO DEL CONTENIDO EN PROFESORES DE BIOLOGÍA: UNA REVISIÓN DOCUMENTAL
}

\section{The Pedagogical Content Knowledge (РСK) in Biology Teachers: A Documentary Review}

\section{O conhecimento pedagógico do conteúdo e um professor de biologia uma revisão documental}

\author{
Guillermo Fonseca Amaya ${ }^{1}$
}

Fecha de recepción: 12 de agosto de 2016

Fecha de aceptación: 17 de mayo de 2017

\section{Resumen}

Este artículo pretende aportar en la comprensión del Conocimiento Pedagógico del Contenido (PCK, por sus siglas en inglés) del profesor de biología, a través de la revisión de las investigaciones publicadas en las bases de datos Eric, Dialnet y SciELO, correspondientes al periodo 2009-2015. Se realizó una revisión documental a través de las siguientes categorías: aproximaciones conceptuales, componentes que lo integran, los procesos metodológicos que se han utilizado para caracterizarlo, la relación entre este tipo de conocimiento y el desarrollo profesional y algunos resultados y conclusiones de las investigaciones. El estudio hace explícito que se mantiene la idea original del pсk propuesto por Shulman, pese a las dificultades que el propio autor ha señalado en la actualidad; con respecto a los modelos de Pck de mayor referencia corresponden a los planteados por Magnusson, Krajcik y Borko (1999), y Park y Oliver (2008; 2008a). Los componentes que con mayor regularidad se presentan en los estudios son el conocimiento de los estudiantes y las estrategias de enseñanza, y la de menor frecuencia es la de currículo. Las investigaciones se fundamentan en procesos de orden cualitativo y cuantitativo, siendo el estudio de caso la metodología que se utiliza con mayor frecuencia, en donde se hace uso de los ReCo, entrevistas y observaciones de clase. En algunas investigaciones se evidencia la articulación entre el estudio del PCK y el desarrollo profesional del profesor de biología.

Palabras clave: PCK; profesor de biología; investigación documental

\section{Abstract}

This article aims to contribute to understanding the Pedagogical Content Knowledge (PCK) of biology teachers by reviewing the research papers published in the Eric, Dialnet and SciELO databases between 2009 and 2015. A documentary review was made under the following categories: conceptual approaches, components, methodological processes used to characterize it, relationship between this type of knowledge and professional development, and some results and conclusions of the investigations. The study makes it clear that the original idea of the PCK proposed by Shulman remains, despite the difficulties pointed out by the author himself; as for the main PCK models, they are the ones proposed by Magnusson, Krajcik and Borko (1999), and Park and Oliver (2008; 2008a). The most frequent components found in the studies are student knowledge and teaching strategies, while the curriculum is

1 Universidad Distrital Francisco José de Caldas, Bogotá, Colombia. Contacto: guifon20@yahoo.com. Este artículo hace parte del desarrollo académico en el Programa de Doctorado Interinstitucional en Educación, Universidad Distrital Francisco José de Caldas, énfasis en Educación en Ciencias, línea de investigación Conocimiento Profesional del Profesor y Conocimiento Escolar. 
the least frequent. The investigations are based on qualitative and quantitative processes, and the case study is the most frequently used methodology, using the CoRe tool, interviews and class observations. Some of the investigations show the articulation between the study of the Pск and the professional development of the biology teacher.

Keywords: Pedagogic Content Knowledge (PCK); biology teacher; documentary revision

\section{Resumo}

Este artigo visa contribuir na compreensão do conhecimento pedagógico do conteúdo (PCK. por sua sigla em inglês) do professor de biologia, através da revisão das pesquisas publicadas nas bases de dados Eric, Dialnet e SciELO, no período de 2009 a 2015. Realizou-se uma revisão documental segundo as seguintes categorias: aproximações conceituais, componentes que o integram, os processos metodológicos que foram desenvolvidos para caracterizá-lo, a relação entre este tipo de conhecimento e o desenvolvimento profissional e alguns resultados e conclusões das pesquisas. O estudo explicita que mantém a ideia original do psk proposta por Schulman apesar das dificuldades que o autor assinalou na atualidade. Ao respeito dos modelos de psk de maior referência, correspondem aos planteamientos de Magnusson, Krajcik e Borko (1999), e Park e Oliver (2008; 2008a). Os componentes que mais frequentemente se apresentam nos estudos são o conhecimento dos estudantes e as estratégias de ensino; e com menor frequência, o currículo. As pesquisas foram fundamentadas em processos de ordem qualitativa e quantitativa, sendo o estudo de caso metodologia utilizada com maior frequência, onde se faz uso dos ReCo, entrevistas e observações de aula. Em algumas pesquisas é evidente a articulação entre o estudo do PCK e o desenvolvimento profissional do professor de biologia.

Palavras-chave: PCK; professor de biologia; pesquisa documental 


\section{Introducción}

El Conocimiento Pedagógico del Contenido se ha constituido en un programa de investigación que aporta en los procesos de formación de profesores y en la educación en ciencias. Así, transcurridos más de 20 años del planteamiento de Shulman, Abell (2008) señala que el Pck se está acercando al estatus de paradigma, que es compartido por toda la comunidad de investigación y que guía nuestro pensamiento sobre el aprendizaje del maestro. Como indica esta autora, aprender a enseñar ciencia no es adquirir una maleta llena de trucos basados en estrategias pedagógicas generales; se trata del desarrollo de un conjunto de conocimientos complejos y contextualizados para aplicar a problemas específicos de la práctica. Además, Abell reconoce que el Pck incluye categorías discretas del conocimiento que son aplicadas sinérgicamente a los problemas de la práctica; este es dinámico; el contenido (la ciencia como materia) es central a él, e involucra la transformación de otros tipos de conocimientos.

En relación con el PCK, es importante reconocer dos periodos en su desarrollo: el primero corresponde a los trabajos de Shulman y sus colaboradores inspirados en el artículo conocimiento y enseñanza: fundamentos de la nueva reforma (1987), y el segundo, a los trabajos derivados de la cumbre sobre este conocimiento, efectuada en Estados Unidos en el año 2012, en la que el propio Shulman realizó algunas consideraciones de su idea original, además de otras observaciones propuestas por investigadores de siete países quienes habían adelantado algunas ampliaciones acerca de este tipo de conocimiento, asunto que se ha publicado en el texto Re-Examing Pedagogical Content Knowledge in Science Education (Berry, Friedrichsen y Loughran, 2015).

Así, en el primer periodo es importante situar la conceptualización que Shulman construyó a través de tres niveles de comprensión acerca del conocimiento profesional del profesor: el primero se refiere a la definición de cuáles son los conocimientos que hacen parte de la formación de un profesor; el segundo al señalamiento de las fuentes que hacen posible su configuración, y el tercero, consiste en plantear un modelo de acción y razonamiento pedagógico, programa que se ha ampliado y reconstruido a partir de diversas investigaciones. Con respecto al conocimiento profesional, Shulman (1987) plantea los siguientes tipos: conocimiento del contenido, conocimiento pedagógico general, conocimiento del currículo, conocimiento pedagógico del contenido, conocimiento de los alumnos y de sus características, conocimiento de los contextos educativos, conocimiento de los objetivos, las finalidades y los valores educativos y de sus fundamentos filosóficos e históricos.
Así, se comienza por reconocer que los profesores poseen un conocimiento profesional singular y que se hace necesario desarrollar investigaciones para comprender la naturaleza y dinámica de cada uno de estos y las interacciones que se pueden establecer entre los tipos de conocimiento. En el marco de la tipología de conocimientos propuestos por Shulman (1987), es fundamental el aporte en relación con la vinculación del conocimiento pedagógico del contenido como un aspecto que caracterizará el conocimiento profesional del profesor.

Entre estas categorías, el conocimiento pedagógico del contenido adquiere particular interés porque identifica los cuerpos de conocimientos distintivos para la enseñanza. "Éste $[\mathrm{PCK}]$ representa la mezcla entre el contenido y la pedagogía en un esfuerzo por comprender cómo un tópico, problema o tema específico es organizado, representado y adaptado para los diversos intereses y habilidades de los estudiantes y presentado para la enseñanza" (Shulman, 1987, p. 8).

Tal conceptualización se constituye en un campo importante de investigaciones en razón que el conocimiento pedagógico de contenido permite comprender los procesos de planificación y actuación del profesor. Por otra parte, Shulman (1987) señala que existen por lo menos cuatro fuentes principales del conocimiento base para la enseñanza: formación académica en la disciplina a enseñar, los materiales y el contexto del proceso educativo institucionalizado, la investigación sobre la escolarización y la sabiduría que otorga la práctica misma.

A propósito de la formación académica en la disciplina a enseñar, Shulman (1987) señala que la primera fuente del conocimiento base es el conocimiento de los contenidos, la comprensión, las habilidades y las disposiciones que deben adquirir los escolares; el autor plantea que frente a este aspecto puede compararse con lo que Schwab (1964, citado en Shulman, 1987; y Shulman, 2015, citado en Berry, Friedrichesen y Loughran, 2015), ha definido como conocimiento de estructuras sustantivas y sintácticas respectivamente. En relación con las estructuras y materiales didácticos, incluye currículos, instituciones con sus jerarquías, organizaciones gremiales de profesores con sus funciones de negociación, los principios, las políticas y las circunstancias de su funcionamiento configuren una importante fuente de conocimiento base. Una tercera fuente es el importante y creciente caudal de bibliografía académica dedicada a la comprensión de los procesos de escolarización, enseñanza y aprendizaje. En estas obras se incluyen las conclusiones y los métodos de investigación empírica en las áreas de docencia, aprendizaje y desarrollo humano, así como los fundamentos normativos, filosóficos y éticos de la educación. La última 
fuente corresponde a la sabiduría adquirida en la práctica: una de las tareas más importantes para la investigación educativa consiste en trabajar junto con los educadores para desarrollar representaciones codificadas de la sabiduría didáctica práctica de los profesores competentes.

En relación con el modelo de acción y razonamiento pedagógico, Shulman (1987) supone la existencia de un ciclo a través de las actividades de comprensión, transformación, enseñanza, evaluación y reflexión. El punto de partida y la culminación del proceso es un acto de comprensión, en donde hace énfasis en la necesidad de la transformación que hace el maestro de su conocimiento para hacerlo comprensible a sus estudiantes, asunto que en el marco de la propia didáctica se constituye en un aspecto fundamental.

Por otro lado, se insiste en la necesidad de continuar desarrollando investigaciones para consolidar teórica y metodológicamente el PCK, con el propósito de superar el estado de pre-ciencia, como lo plantea Abell (2007), al igual que Van Driel, Verloop y de Vos (1998). En este misma perspectiva, Park y Oliver (2008) señalan la necesidad de continuar trabajando sobre este conocimiento en razón de su naturaleza amorfa causa dificultad en su uso explícito como una herramienta conceptual; en otras palabras, ha sido difícil construir una imagen clara, no solo de cómo se desarrolla el andamiaje de este conocimiento en los docentes, sino también de cómo evaluarlo una vez construido. Al respecto, Fischer, Borowski y Tepner (2012) expresan que

la mayor parte de la investigación sobre la ск у Рск, que son las facetas más importantes de conocimiento profesional sobre la educación científica (didáctica de la ciencia o la didáctica de las disciplinas científicas diferentes), todavía se mantiene en un nivel descriptivo. Por otra parte, estudios realizados recientemente no se han referido suficientemente unos a otros, generando un déficit en cuanto a la fiabilidad y la validez de sus resultados. (Citado en Fraser, Tobin y McRobbie, 2012, p. 443, traducción propia).

Respecto a la segunda etapa del desarrollo del pck, Shulman (2015) plantea:

Cualquier idea debe ser entendida como una contribución a la conversación de la cual hace parte pero no como una verdad universal o generalización. El PCK ciertamente tiene debilidades y confió que muchos de ustedes están apuntalando aquellas deficiencias, elaborando, yendo más allá de la formulación inicial como debe ser el destino de cualquier buena idea razonable. (Citado en Berry, Friedrichesen y Loughran, 2015, p. 4, traducción propia).

Sobre las limitaciones, señala Shulman que la primera corresponde a la insuficiencia de emoción, afecto, sentimientos y motivación, además de la poca atención al carácter moral de la enseñanza y la reducida comprensión del papel del profesor en la construcción de ciudadanos en una sociedad democrática. La segunda limitación corresponde a una comprensión de este conocimiento con énfasis en el orden intelectual, con una preocupación más centrada en la mente, conocimiento, razonamiento pedagógico, que la practica misma; así se profundizó en el constructo de Pck y menos en su relación con la practica pedagógica del maestro. Por otra parte, se reconoce como una tercera limitante la poca atención a la influencia del contexto socio cultural donde se desarrolla la práctica y en consecuencia donde se desarrolla e implementa el pck. En cuarto lugar, corresponde a la no comprensión entre la configuración y desarrollo de este tipo de conocimiento y los diversos aprendizajes de los estudiantes de orden conceptual y emotivo (Shulman, 2015, citado en Berry, Friedrichesen, y Loughran, 2015).

El PCK se ha constituido en un tipo del conocimiento sobre el cual se han desarrollado diversos aportes en relación con su comprensión teórica y metodológica y ha sido objeto de diversas investigaciones en el mundo. A continuación, se describen los aportes de algunos de los investigadores que han marcado el desarrollo de posteriores investigaciones, constituyéndose en un referente fundamental en esta línea de trabajo. Entre otros es importante citar los trabajos Gess-Newsome (1999; 1999a), Magnusson, Krajcik y Borko (1999) y Park y Oliver (2008); asimismo, Gess-Newsome (2015), citado en Berry, Friedrichsen y Loughran (2015), en razón a que se han constituido en referentes para desarrollar investigaciones en este campo.

Gess-Newsome (1999) plantea esencialmente dos modelos de pck: el integrado y el de transformación. En los dos modelos las componentes son: conocimiento de la materia (SMK), conocimiento pedagógico ( $\mathrm{PK}$ ) y conocimiento contextual (ck), respecto al primer modelo (integrado), las componentes no funcionan de manera independiente, sino que se interrelacionan para formar ese constructo de saberes necesarios en el Pck. La autora reconoce que un factor limitante es que el profesor retrasmita modelos de enseñanza o se centre solo en una de las componentes. En el segundo modelo (transformación), los componentes funcionan de manera separada, pero al integrarse con los otros componentes se transforma en el PCK. 
En un extremo, el Pck no existe, y el conocimiento del profesor puede ser explicado fácilmente por la intersección de los tres constructos: conocimiento de la materia, conocimiento pedagógico y conocimiento contextual. La enseñanza, entonces, es un acto de conocimiento integrado a través de esos tres dominios, por conveniencia lo llamare modelo integrativo. En el otro extremo, el Pck es la síntesis, de todo el conocimiento necesario con el fin de ser un profesor efectivo. En este caso, el pck es la trasformación de los tres constructos. En una forma única -la única forma de conocimiento que impacta la práctica docente. Lo llamaré modelo transformativo. (Gess-Newsome, 1999, p. 10).

Magnusson, Krajcik y Borko (1999) plantean que la característica definitoria de conocimiento pedagógico de contenido es su conceptualización como resultado de una transformación del conocimiento, así este se conceptualiza como el conocimiento del contenido pedagógico para la enseñanza de la ciencia como un conjunto de cinco componentes: a) orientación hacia la enseñanza de las ciencias; b) el conocimiento y creencias acerca del currículo de ciencias; c) conocimientos y creencias sobre los estudiantes acerca de la comprensión de temas específicos de la ciencia; d) conocimientos y creencias acerca de la evaluación de la ciencia, y e) conocimientos y creencias acerca de las estrategias de instrucción para la enseñanza de ciencias. En este modelo, los cinco componentes se presentan en una forma lineal con énfasis en la interacción única entre las orientaciones de enseñanza de las ciencias y cada uno de los otros cuatro componentes y en consecuencia se ignora la interacción que se sucede entre ellos.

Park y Oliver (2008a) lo definen como una integración de cinco componentes representados en el modelo pentágono: a) Orientaciones didácticas para los docentes de Ciencias; b) Conocimiento de comprensión de los estudiantes en Ciencias; c) Conocimiento del Plan de Estudios de Ciencias; d) Conocimiento de estrategias de enseñanza y Representaciones, y e) Conocimiento de Evaluación del aprendizaje de la Ciencia. El modelo presenta los componentes en una forma pentagonal para enfatizar la interrelación entre ellos y poner el mismo peso en cada interacción; a este respecto, un aumento en un solo componente, sin un aumento correspondiente en los otros, no puede ser suficiente para cambiar toda la estructura PCK para estimular cambios en la práctica, la integración entre los componentes es posible a través de procesos de reflexión en y sobre la acción sucedida en la práctica pedagógica.

\section{Metodología}

El artículo se deriva de un proceso metodológico inscrito en los planteamientos del enfoque cualitativo, en singular, desde la revisión documental, que de acuerdo con Latorre, Rincón y Arnal (2003), se constituye como el proceso dinámico que consiste esencialmente en la recogida, clasificación, recuperación y distribución de la información. Uribe (2011), citado en Paramo (2011), plantea que la investigación documental es en esencia el estudio metódico, sistemático y ordenado de datos, y documentos, los cuales una vez recopilados, contextualizados, clasificados, categorizados y analizados, sirven de base para la comprensión del problema.

Desde este lugar de comprensión metodológico, el proceso investigativo se desarrolló a través de cuatro fases: la primera, búsqueda en la base de datos (Eric, Dialnet y SciELO) y la selección de los artículos, a través de las palabras clave (PCK, profesor de biología); en ese sentido, las bases de datos objeto de búsqueda se seleccionaron con el criterio de uso por parte de la comunidad de investigadores y de acceso libre para obtener la información.

La segunda fase correspondió a la elaboración y aplicación de una ficha analítica de lectura a los artículos seleccionados, en la que se incluían las propias categorías de análisis derivadas del campo conceptual (aproximaciones conceptuales, sus componentes, los procesos metodológicos que se han utilizado para caracterizarlo, la relación entre el PCK y el desarrollo profesional, algunos resultados y conclusiones de las investigaciones). El proceso se realizó a través de la lectura cruzada entre los miembros del grupo de investigación; así, cada artículo fue objeto de interpretación por pares, quienes presentaron cada ficha en sesiones de trabajo para señalar los asuntos convergentes y divergentes y tomar decisiones acerca de los hallazgos.

La tercera fase correspondió a la confrontación de los hallazgos por parte del grupo de investigación para cada una de las categorías, que se realizó a través del diseño de una matriz en el programa Excel, en donde se ubicaban para cada artículo y para cada categoría los consensos planteados en el grupo; la cuarta fase corresponde a la construcción del texto final con el fin de articular los hallazgos con los marcos de referencia para cada una de las categorías. Este proceso aporta, como los señala Uribe (2011), en la comprensión del problema objeto de análisis, que para este caso corresponde a una mirada respecto a los desarrollos del conocimiento pedagógico del contenido en profesores en biología. 


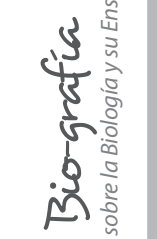

\begin{tabular}{|c|c|c|c|c|c|c|c|c|c|}
\hline 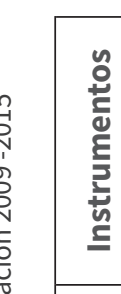 & 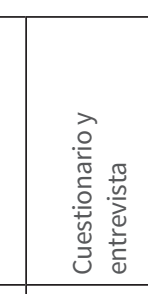 & 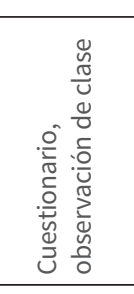 & 敦 & 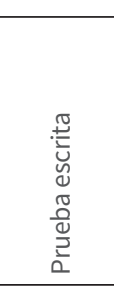 & & 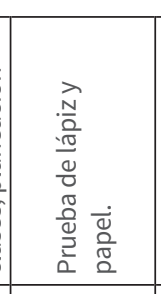 & & 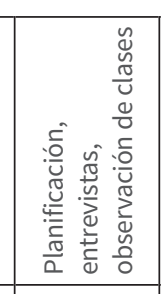 & 噌 \\
\hline 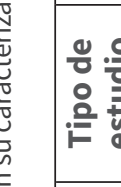 & & & & & & & & 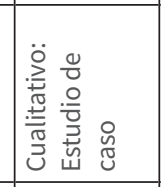 & \\
\hline 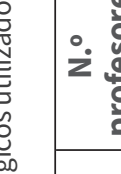 & & & & $\underset{N}{ \pm}$ & & $\stackrel{\mathscr{g}}{g}$ & & & \\
\hline$\overline{\frac{a}{z}}$ & 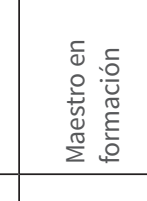 & 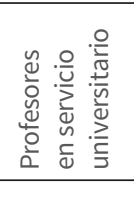 & 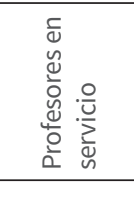 & & 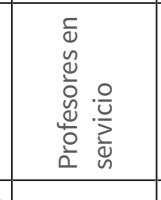 & 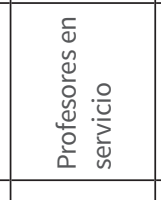 & & 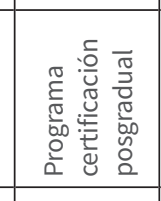 & \\
\hline 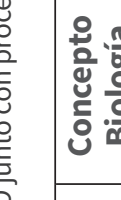 & & 亳 & & & 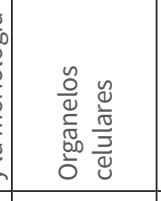 & 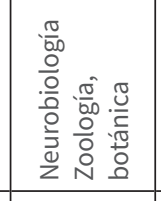 & 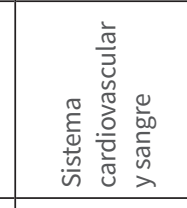 & & \\
\hline 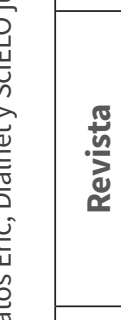 & & 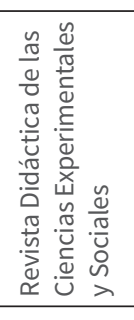 & 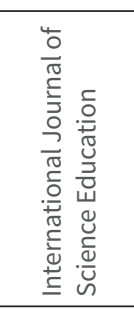 & 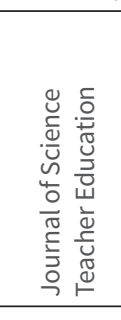 & 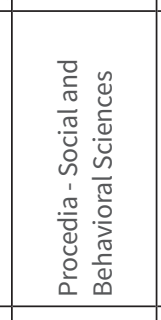 & 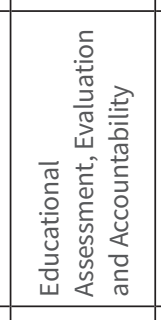 & 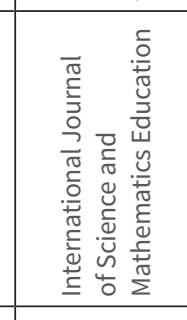 & 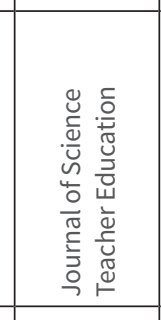 & 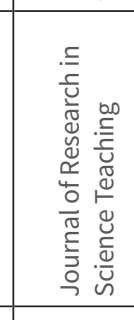 \\
\hline$\frac{n}{0}$ & & & & & 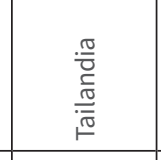 & 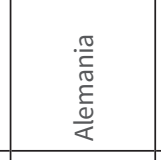 & & 总 & 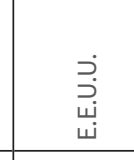 \\
\hline 旅 & 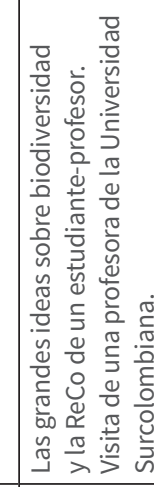 & 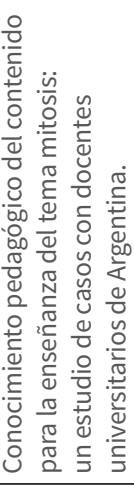 & & 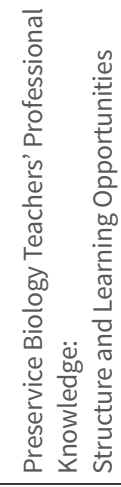 & 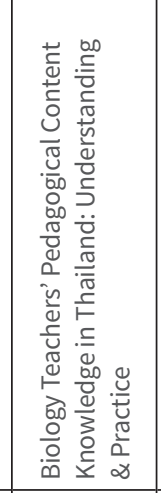 & 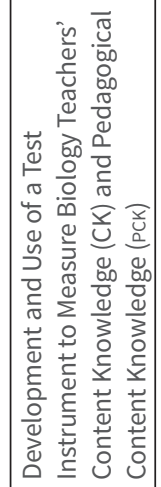 & 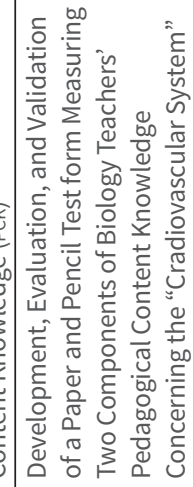 & 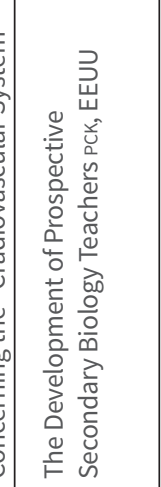 & 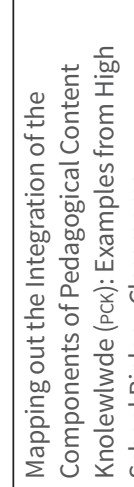 \\
\hline$\frac{\circ}{8}$ & 总 & 䜌 & 商 & 㒚 & 喜 & 誉 & $\frac{\mathrm{m}}{\mathrm{z}}$ & 伝 & 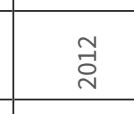 \\
\hline 高 & 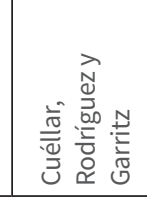 & 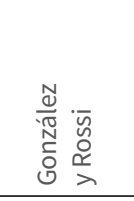 & & & 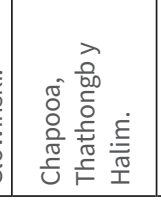 & 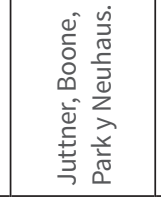 & 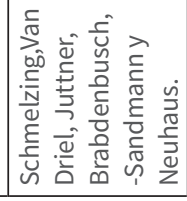 & 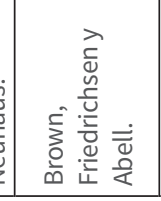 & 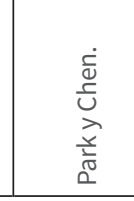 \\
\hline
\end{tabular}




\begin{tabular}{|c|c|c|c|c|}
\hline 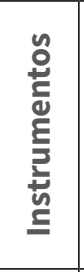 & 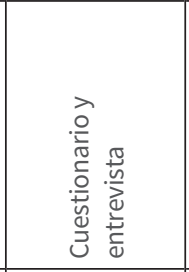 & 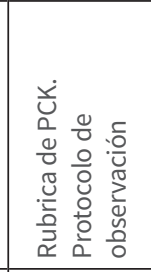 & 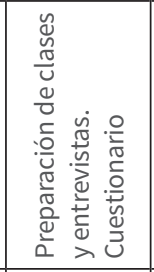 & 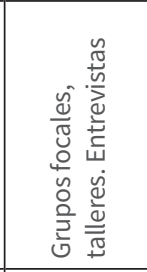 \\
\hline 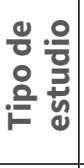 & 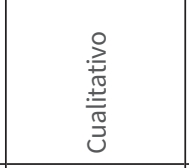 & 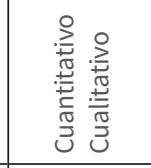 & 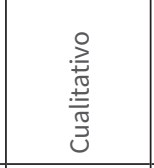 & 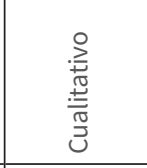 \\
\hline 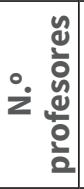 & N & $\Lambda$ & ㄱ & 옹 \\
\hline$\stackrel{\Xi}{\Sigma}$ & 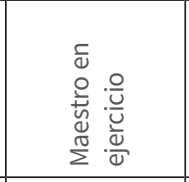 & 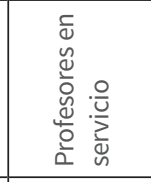 & 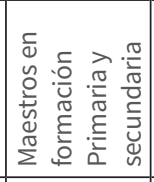 & 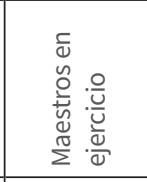 \\
\hline 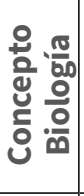 & 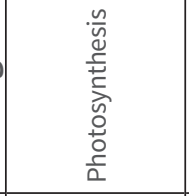 & 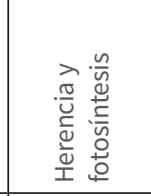 & 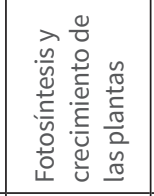 & $\frac{\frac{\pi}{3}}{\frac{\pi}{\pi}}$ \\
\hline 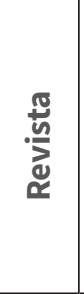 & 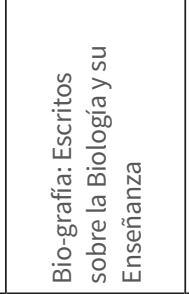 & 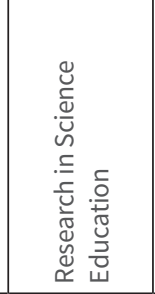 & 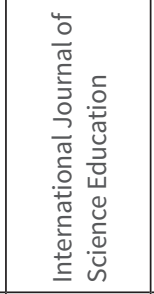 & 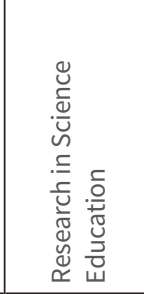 \\
\hline$\frac{n}{d x}$ & $\begin{array}{l}\frac{\pi}{0} \\
\frac{\bar{g}}{\bar{E}} \\
\frac{0}{0} \\
\end{array}$ & نْن & 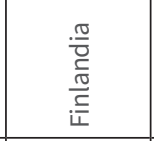 & $\begin{array}{l}\bar{v} \\
\underline{\underline{w}} \\
\end{array}$ \\
\hline 올 & 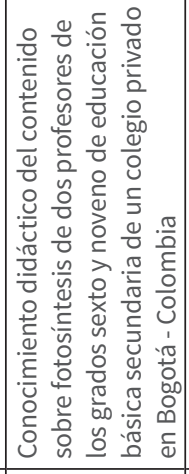 & 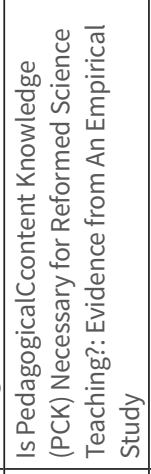 & 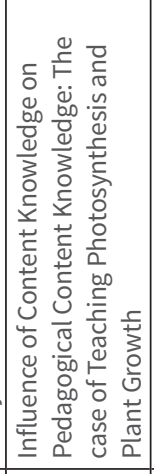 & 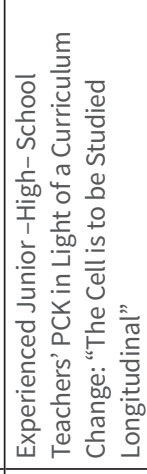 \\
\hline 异 & $\underset{\sim}{\tilde{N}}$ & $\overrightarrow{\text { त् }}$ & ণ্ & ষ্ণ \\
\hline $\begin{array}{l}\grave{0} \\
\stackrel{+}{z}\end{array}$ & 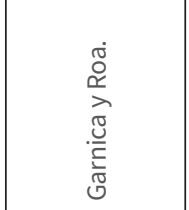 & 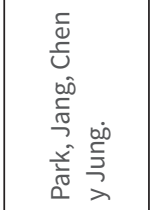 & 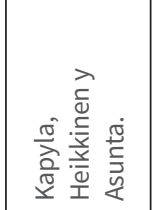 & 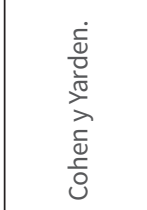 \\
\hline
\end{tabular}

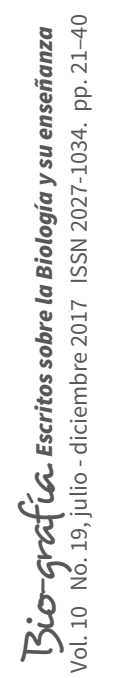




\section{Acerca de las conceptualizaciones de PCK en las investigaciones reportadas}

El estudio reporta de manera general que este conocimiento es objeto de investigación en América, Europa y Asia, contribuyendo a la comprensión de los procesos de formación tanto en maestros en formación como en ejercicio profesional. Los contenidos sobre los cuales se hacen los estudios corresponden a biodiversidad, mitosis, genética, evolución, microbiología, organelos celulares, neurobiología, zoología, botánica, sistema cardiovascular, herencia y fotosíntesis. Se evidencia además que en las investigaciones se privilegia un tratamiento de orden cualitativo, y la muestra de los trabajos varía desde el trabajo con uno hasta 274 maestros.

En la conceptualización, en las investigaciones realizadas con profesores de biología, se evidencia que se sustentan en los planteamientos originales de Shulman (1986-1987) y los aportes Gess-Newsome (1999), en relación con la idea de los modelos de рск integrativo y transformativo.

En este asunto es importante actualizar la idea de los modelos debido a la dificultad de demarcar la transformación o la integración en la actuación de los docentes. Gess-Newsome, citado en Berry, Friedrichsen y Loughran (2015), plantea que "en [la] introducción al libro рск 1999 (Gess-Newsome, 1999a), discuto la idea de Pck como producto de la integración o transformación, sin embargo, sobre la base de nuestras conversaciones en la Cumbre del PcK, considero que esta distinción ya no es útil” (p. 40). En este mismo sentido, Kind (2015), citado en Berry, Friedrichsen y Loughran (2015) señala la noción de PCK como transformador o integrador se deja caer a favor de una amplia gama de posibles componentes que interactúan.

Además de situar la conceptualización en los modelos de integración o transformación, las investigaciones se refieren a los componentes que lo constituye; sin embargo, respecto a su conceptualización en las investigaciones en el campo de los profesores de biología es importante señalar que no se avanza en la comprensión epistemológica de este conocimiento, es decir, respecto a su naturaleza de constitución, de validación y del carácter práctico en tanto que es un conocimiento que se resuelve en la acción no es objeto de problematización en estas investigaciones, en este sentido no se explora la idea de la sabiduría en la práctica que plantea Shulman.

\section{Acerca de las componentes del PCK en las investigaciones reportadas}

El PCK se ha constituido en un tipo de conocimiento que aporta en la comprensión de lo que sucede en los procesos de enseñanza de un tópico específico; en este sentido, su descripción se desarrolla a través de la caracterización de sus componentes.

Tabla 2. Componentes del Pck que se incluyen en las investigaciones objeto de la revisión documental

\begin{tabular}{|c|c|c|c|c|c|c|c|c|}
\hline \multirow{2}{*}{ Autor } & \multirow{2}{*}{ Año } & \multicolumn{7}{|c|}{ Componente } \\
\hline & & 1. & 2. & 3. & 4. & 5. & 6. & 7. \\
\hline González y Rossi. & 2015 & $\mathrm{x}$ & $x$ & $\mathrm{x}$ & & $x$ & & \\
\hline Mthethwa-Kunene, Onwu y Villiers. & 2015 & $\mathrm{x}$ & $x$ & $\mathrm{x}$ & & & & \\
\hline Cuéllar, Rodríguez y Garritz. & 2015 & $x$ & & $\mathrm{x}$ & & & & \\
\hline Großschedl, Harms; Kleickmann y Glowinski. & 2015 & & $x$ & $\mathrm{x}$ & $x$ & $\mathrm{x}$ & & \\
\hline Chapooa, Thathongb y Halim. & 2014 & $x$ & $x$ & $\mathrm{x}$ & $x$ & $x$ & & \\
\hline Juttner, Boone, Park y Neuhaus. & 2013 & & $\mathrm{x}$ & $\mathrm{x}$ & & & & \\
\hline Schmelzing, Van Driel, Juttner, Brabdenbusch, Sandmann y Neuhaus. & 2013 & & $x$ & $\mathrm{x}$ & & & & \\
\hline Brown, Friedrichsen y Abell. & 2012 & $\mathrm{x}$ & $x$ & $\mathrm{x}$ & & & & \\
\hline Garnica y Roa. & 2012 & $x$ & $x$ & $\mathrm{X}$ & $x$ & $x$ & $x$ & $x$ \\
\hline Park y Chen. & 2012 & $\mathrm{x}$ & $\mathrm{x}$ & $\mathrm{x}$ & $\mathrm{x}$ & $\mathrm{x}$ & & \\
\hline Park, Jang; Chen y Jung. & 2011 & & $\mathrm{x}$ & $x$ & & & & \\
\hline Kapyla, Heikkinen y Asunta. & 2009 & $x$ & $x$ & $\mathrm{x}$ & $x$ & & & \\
\hline Cohen y Yarden. & 2009 & $x$ & $x$ & $x$ & $x$ & $x$ & & \\
\hline
\end{tabular}

Nota: En la tabla los componentes de PCK se encuentran numeradas de la siguiente manera: 1. Orientaciones; 2 . Estrategias; 3. Conocimiento de los estudiantes; 4. Currículo; 5. Evaluación; 6. Contenido disciplinar; 7. conocimiento experiencial. 
En el trabajo de Park, Jang, Chen y Jung (2011) señalan que el instrumento de medición del PCK utilizado en el estudio se centra en dos de sus componentes: el conocimiento de la comprensión del estudiante y el conocimiento de estrategias de enseñanza. El resultado de este estudio revela que estos dos componentes se relacionan positivamente con la orientación hacia la reforma de la instrucción. El hallazgo de esta investigación promueve centrar los esfuerzos de cualifiación docente en comprender el tratamiento de las concepciones de los estudiantes y su relación con las dificultades de aprendizaje. Por otra parte, plantea la necesidad de pensar los componentes del pck de manera integrada y de ampliar la comprensión de las relaciones que se suceden entre todas los componentes que hacen parte de este: la mayoría de los investigadores están de acuerdo con los dos componentes clave de Shulman, es decir, el conocimiento de la comprensión del estudiante y el conocimiento de estrategias de enseñanza (Park y Oliver, 2008a). Estamos de acuerdo en que la medición de solo dos componentes es una limitación del instrumento, pero este intento debe ser un primer paso importante para desarrollar una medida más inclusiva de PCK dado que la construcción de este es en sí mismo aún no se entiende en su totalidad (Park, Jang, Chen y Jung, 2011, p. 255).

En el estudio realizado por Parky Chen (2012) se examinó la naturaleza del proceso a través del cual los cinco componentes constituyentes se integraron. En primer lugar, este estudio proporciona apoyo empírico para el tema de la especificidad, que ha llegado a un acuerdo entre los investigadores en este campo. Aunque muchas investigaciones indican el tema de la especificidad de PCK, se sabe poco acerca de lo que contribuye a esta naturaleza. Los resultados de este estudio sugieren que el tema en particular no solo depende de los componentes que constituyen PCK de un maestro para un tema en particular, sino también sobre cómo y en qué medida esos componentes interactúan entre sí. Esto implica que este conocimiento es más que la suma de los componentes constituyentes y que la interacción sinérgica entre los elementos, además de la cantidad de conocimiento en componentes individuales que contribuye a la calidad de PCK (Abell, 2008).

Así, Park y Chen (2012) reiteran empíricamente la afirmación que los componentes son: el conocimiento de la comprensión del estudiante y el conocimiento de estrategias de enseñanza y representaciones, que son fundamentales en la formación de la estructura del PCK de un profesor. Por ejemplo, los profesores deben tener la posibilidad de analizar los conceptos erróneos y dificultades de los estudiantes en el aprendizaje de un tema en particulary luego se conecten los resultados del análisis a la práctica. Esta sugerencia ha sido apoyada también por otros estudios empíricos (por ejemplo, Clermont, Krajcik y Borko, 1993; Van Driel et al., 1998). Por otra parte, en el estudio de Park y Chen (2012) se proporciona evidencia empírica donde se explica la relación entre las orientaciones y los otros componentes. A pesar de que la idea de las orientaciones en la enseñanza de la ciencia actúan como filtro en la configuración del contenido y el desarrollo de los otros componentes (Friedrichsen et al., 2011), muy pocos estudios empíricos han investigado las interacciones entre las orientaciones y otros componentes de este. Al considerar la importante función de la orientación de la enseñanza de la ciencia como un "mapa conceptual" (Grossman, 1990, p. 86), con la que los profesores toman decisiones de instrucción sobre objetivos de la lección, estrategias de enseñanza, la selección de materiales curriculares, el contenido y métodos de evaluación de los alumnos y tareas de los estudiantes (Borko y Putnam, 1996), se necesitan más estudios empíricos para entender las orientaciones enseñanza de la ciencia en relación con otros componentes Pck y para toda la construcción de este en el contexto de la práctica docente.

Además, Park y Chen (2012) plantean que otro patrón notable en la síntesis de los componentes en el PCK fue que el conocimiento curriculo de ciencias tenía la conexión más limitada con otros componentes. Del mismo modo, señalan que el conocimiento de la evaluación se incorporó con poca frecuencia en el Pck de los maestros. Sin embargo, cuando se integró, se suele conectar con el conocimiento de estrategias de enseñanza y las representaciones y conocimiento de la comprensión del estudiante. Esta conexión sugiere que cuando los maestros tomaron en consideración la evaluación, era probable alinear la evaluación con el aprendizaje y métodos de enseñanza, que son aspectos críticos de la enseñanza eficaz (National Research Council, 1996). Dado que el conocimiento de estrategias de enseñanza y representaciones y conocimiento de la comprensión del estudiante hacen más conexiones con otros componentes, una inferencia lógica sería que un aumento en la evaluación estimulará su conexión con estos componentes y que a su vez provocara una fuerte conexión y coherencia entre los componentes. Teniendo en cuenta la importancia de la evaluación en la interacción entre los componentes, se deben hacer más esfuerzos de investigación para examinar la forma en que la evaluación es sintetizada en PCK y cómo mejorar esta en sí y sus conexiones con otros componentes.

Park, y Chen (2012) señalan, en general, que los resultados de este estudio convergen en el punto de la síntesis de los componentes, que no es un proceso sencillo relacionado principalmente con la simple posesión de esos elementos. Por el contrario, las construcciones de PCK 
son en gran medida influenciadas por la interacción de los diferentes componentes (Hashweh, 2005). No siempre es el caso que un aumento en uno de los componentes mejora simultáneamente la integridad y funcionamiento de todo el PCK. La falta de coherencia entre los componentes puede ser problemático en el desarrollo de este conocimiento. Además, un mayor conocimiento en un solo componente puede no ser suficiente para estimular el cambio en la práctica. En este sentido, para ayudar a los maestros a desarrollar este conocimiento, se debe hacer énfasis no solo en la cantidad de conocimientos, en componentes constituyentes individuales, sino en el crecimiento de la conectividad y la complejidad del PcK en su conjunto.

Brown, Friedrichesen y Abell (2012) plantean la necesidad de reconsiderar el uso del modelo pck; Magnusson et al. (1999) consideran que son necesarios modelos más elaborados para tener en cuenta la integración de los componentes del conocimiento con el fin de comprender mejor cómo los profesores aprenden a enseñar ciencia. Un modelo pck en desarrollo debe ser flexible y fluido, no tratar el conocimiento a partir de componentes fragmentados. Hemos aprendido del análisis de los datos que los profesores de ciencias de secundaria adquieren más experiencia en la interacción que se establece entre el conocimiento de los profesores sobre sus alumnos y el conocimiento de las secuencias de instrucción se vuelve más integrado. Es necesario más estudios para proporcionar una visión más completa del conocimiento de las ciencias por parte de los maestros de secundaria en relación con orientaciones, el conocimiento de los alumnos y el conocimiento de las secuencias de instrucción, para un cierto grupo de estudiantes en un contexto particular, generando procesos de integración entre los componentes en PCK en un manera coherente (Loughran, Berry y Mulhall, 2006; Van Driel y Verloop, 2002). La investigación indica que estos componentes interactúan de maneras complejas (Park y Oliver, 2008a), y una integración coherente entre ellos es fundamental para el desarrollo de este conocimiento en la práctica (Cochran, King y DeRuiter, 1991; Magnusson, Krajcik, y Borko, 1999).

Juttner, Boone, Park, y Neuhaus (2013) abordan el conocimiento de las estrategias de enseñanza y su papel en las lecciones y el conocimiento de comprensión de los estudiantes. Estos componentes (desde la dimensión declarativa, para el componente "conocimiento acerca de la comprensión de los estudiantes") acuden a la revisión de otras investigaciones a pesar de las diferentes conceptualizaciones de PCK (Abell, 2007; Park et al., 2011; Baumert et al., 2010; Baxter y Lederman. 1999, Berry et al., 2008; Blomeke et al., 2008; Hashweh, 2005; Hill et al., 2005; Magnusson et al., 1999). Por otro lado, existe un acuerdo con respecto a dos aspectos críticos del Pck: conocimiento de la comprensión de los estudiantes y el conocimiento de las estrategias de enseñanza (Park y Oliver, 2008; Baumert et al., 2010; Lee y Luft, 2008; van Driel et al., 1998).

Schmelzing et al. (2013) desarrollan la investigación a través de dos componentes: conocimiento acerca de las concepciones y aprendizaje de los estudiantes y el conocimiento de las representaciones y estrategias; estos componentes se cruzan en el pck de tipo declarativo para el primer componente, $y$ de tipo procedimental para el segundo.

En el trabajo de Cohen y Yarden (2009) se asume el modelo de Magnusson et al. (1999) como eje orientador de la investigación, en el que se conceptualiza el Pck como un conjunto de cinco componentes; el uso de las estrategias está influida por las creencias o falta de Pck y el conocimiento de los contenidos (Magnusson et al., 1999). Los maestros ocupan su tiempo en las dificultades de comprensión de los estudiantes, lo que hace que se reduzca el tiempo dedicado al desarrollo del concepto, aunque el proceso mismo de interés que tiene el docente por hacer que el estudiante comprenda constituye en sí mismo parte de la clase y del PCK.

Kapyla, Heikkinen, y Asunta (2009) trabajan los componentes de pck inmersos en el modelo de Magnusson et al. (1999): los problemas conceptuales de los estudiantes, el contenido básico de la enseñanza (el conocimiento del plan de estudios), el conocimiento de los métodos de enseñanza, el conocimiento de los métodos de evaluación de contenido específico y orientaciones para la enseñanza de la ciencia. De estos, los métodos de evaluación no se incluyeron en el estudio porque en la enseñanza no se observó y ninguno de los participantes incluyó la evaluación de sus planes de estudio.

Mthethwa-Kunene, Onwu y De Villiers (2015) abordaron para el estudio realizado tres componentes del PCK del modelo de Magnusson et al. (1999): el conocimiento de la materia por parte del maestro, el conocimiento pedagógico, conocimiento de las preconcepciones y las dificultades de aprendizaje de los estudiantes, donde los autores reconocen que el pck es una forma compleja de conocimiento construido por los propios profesores (Mthethwa-Kunene, Onwu y De Villiers, 2015, p. 23).

Großschedl, Harms, Kleickmann y Glowinski (2015) tuvieron en cuenta cuatro componentes, según Tamir (1988): conocimiento de estrategias de instrucción para la enseñanza, conocimiento de la comprensión de los estudiantes y conocimiento curricular y conocimiento de la evaluación. Se midió por separado CK, PCK y PK. 
González y Rossi (2015) en su estudio abordó cuatro de las componentes de modelo de Magnusson et al. (1999): Orientación en la enseñanza, comprensión por parte de los estudiantes, conocimiento de estrategias instruccionales y conocimiento de la evaluación de la comprensión de los estudiantes.

Cuellar, Rodríguez y Garritz (2015) trabajan dos componentes del modelo Magnusson et al. (1999): las orientaciones hacia la enseñanza de la ciencia el conocimiento de la finalidad y objetivos para la enseñanza de la ciencia en un grado en particular y conocimientos y creencias acerca de la comprensión de los estudiantes sobre temas científicos específicos. En el artículo de investigación no se hace explicito que los dos componentes del PcK anteriores se privilegien; sin embargo, las preguntas utilizadas en el cuestionario podrían hacer pensar que se apoyan en los componentes de Magnusson et al. (1999).

Chapoo, Thathong, y Halim (2014) desarrollan las cinco componentes del modelo Magnusson et al. (1999): orientaciones hacia la enseñanza de las ciencias, el conocimiento del plan de estudios, el conocimiento de evaluación, el conocimiento de los estudiantes la comprensión de la ciencia, el conocimiento de las estrategias de instrucción, donde los autores reconocen que los profesores privilegian las componentes de orientaciones hacia la enseñanza de las ciencias y el conocimiento de evaluación; con esto, se descuida buena parte del componente del conocimiento de los estudiantes en torno a la comprensión de la ciencia.

Garnica y Roa (2012) trabajan en particular ocho componentes recopilados de varios modelos (Charrier, 2005, 2009; Loughran et al., 2001;Valbuena, 2007), entre ellos: conocimiento acerca de los estudiantes, conocimiento acerca del contenido disciplinar, formas de evaluar el aprendizaje del concepto, estrategias didácticas para enseñar el concepto, propósitos de la enseñanza del concepto, dificultades y limitaciones en la enseñanza del concepto, currículo y conocimiento experiencial del profesor. El componente "propósitos de la enseñanza y dificultades de la enseñanza" está inmerso en los componentes de conocimiento de las orientaciones y conocimiento de los estudiantes respectivamente. Los autores abordan y analizan todos los componentes sin darle más relevancia a uno que al otro; por esto, se discuten aquellas donde el docente se encuentra con mayor dificultad, por ejemplo, el componente "estrategias didácticas para enseñar el concepto", donde las dos maestras investigadas tienen poco conocimiento de la historicidad del concepto y la historia de su enseñanza.
Sobre los componentes del Pск que se presentan con mayor regularidad en las investigaciones corresponden a las estrategias de enseñanza y el conocimiento de los estudiantes (Cuellar, Rodríguez y Garritz, 2015; González y Rossi, 2015; Mthethwa-Kunene, Onwu y de Villiers, 2015; Chapoo, Thathong y Halim, 2014; Park y Chen, 2012; Garnica y Roa, 2012; Brown, Friedrichesen y Abell, 2012; Cohen y Yarden, 2009; Käpyläa, Pekka Heikkinenb y Asuntaa, 2009; Park y Oliver, 2008a; Park y Chen, 2012; Park, Jang, Chen y Jung, 2011).

Finalmente, con respecto al componente que presenta menor reconocimiento por parte de los maestros en la configuración del PCK, se encuentra el componente del currículo (Großschedl, Harms, Kleickmann y Glowinski, 2015; Chapoo, Thathong y Halim, 2014; Park y Chen, 2012; Garnica y Roa, 2012; Cohen y Yarden, 2009; Käpyläa, Pekka Heikkinenb y Asuntaa, 2009). Sobre el componente de evaluación, puede consultarse González y Rossi (2015); Großschedl, Harms, Kleickmann y Glowinski (2015); Chapoo, Thathong y Halim (2014); Park y Chen (2012); Garnica y Roa (2012); Cohen y Yarden (2009).

\section{Acerca de los procesos metodológicos en las investigaciones}

Acerca de los procesos metodológicos que se utilizan en las investigaciones, estas se caracterizan por dos tendencias: la primera, desde una racionalidad en donde se privilegia métodos de orden cuantitativo y generalista para dar cuenta del PCK de los profesores, para lo cual se diseñan instrumentos a través de índices de confiabilidad que garantizan los hallazgos obtenidos. En este tipo de estudios es el Pck declarativo el que se privilegia en razón de la propia naturaleza del estudio; en esta tendencia se pueden situar los trabajos de Großschedl, Harms, Kleickmann y Glowinski (2015). En dicho estudio se incluyeron 274 profesores en formación de diferentes universidades alemanas, en el que se midió el de manera cuantitativa por medio de pruebas escritas el CK, PCK y PK de los participantes. El cK (conocimiento del contenido) se evaluó mediante 78 ítems, con relación a los conceptos de evolución (34), Genética y Microbiología (21), y la morfología (23). El pck se evaluó mediante 66 ítems, con relación al conocimiento de la comprensión de los estudiantes (18), conocimiento de estrategias (31), el conocimiento de evaluación (7), y el conocimiento del plan de estudios de biología (10); el diseño del instrumento fue realizado por biólogos y educadores. El pK (Conocimiento Pedagógico) se evaluó mediante 36 ítems, con relación al 
conocimiento de la comprensión de los estudiantes (16) y el conocimiento de las estrategias (20), los casos fueron analizados por el programa MPlus 5.21., donde se midió de manera separada cK, РсK y PK; sin embargo, los autores señalan que estos se correlacionan.

Juttner, Boone, Park y Neuhaus (2013) examinan el desarrollo y uso de instrumentos para medir la ск y Рск profesores de biología. En particular, este estudio describe un método para desarrollar instrumentos fiables, objetivos y válidos de medición de estos conocimientos en los profesores, en cuatro pasos con el uso de datos empíricos. Además, el estudio explora si estos conocimientos pueden ser medidos como categorías separadas de conocimiento mediante el uso de una prueba escrita. Se presenta un modelo teórico que guía el desarrollo de pruebas y proporciona los pasos para desarrollar y validar los instrumentos. También se proporcionan detalles sobre el cálculo de las calificaciones medidas a escala Rasch para 158 profesores de biología. Los resultados indican que los instrumentos miden ск у PсK en los maestros de una manera objetiva, válida y confiable, esto sugiere que los nuevos instrumentos se pueden utilizar en combinación con observaciones en el aula para examinar la calidad de la enseñanza y aún más su relación con el aprendizaje de los estudiantes. El estudio se realizó en tres temas: neurobiología, zoología y botánica.

Schmelzing, van Driel, Juttner, Brabdenbusch, Sandmann y Neuhaus (2013) presentan un estudio sobre el desarrollo, la evaluación y validación de una prueba escrita para medir PCK declarativo de profesores de biología en el tema de la sangre y del sistema cardiovascular humano. El desarrollo de la prueba se basa, en una revisión de la literatura de investigación sobre PCK y un análisis de 50 lecciones de biología en video. El instrumento de la prueba final se compone de 15 ítems distribuidos en 2 escalas. El estudio se realizó con 93 profesores en formación y profesores de biología en servicio y 12 biólogos, lo que confirma que esta medida de pck declarativa de profesores de biología era fiable, objetiva y válida.

Park, Jang, Chen y Jung (2011) utilizaron un método de investigación cuantitativa para investigar la correlación entre el nivel de PCK, medida por la rúbrica de (Park et al., 2008) y el grado en que en su clase se desarrolla (Sawada et al., 2002). Los datos incluyen 33 sesiones de instrucción de la fotosíntesis y la herencia, grabada con 7 profesores de biología de una escuela secundaria. Cada sesión se le dio una calificación en tanto la rúbrica de PCK y RTOP por dos evaluadores independientes. La rúbrica se compone de nueve elementos que se pretenden representar el conocimiento de la comprensión de los estudiantes (KSU) y las estrategias de instrucción (KISR) a través de tres etapas de formación diferentes: la planificación, la implementación y la reflexión; cada elemento se calificó mediante una escala de cuatro puntos desde 1, "limitada" a 4, "ejemplar" para una posible puntuación total de 36 para el instrumento en su conjunto. La consistencia interna, según las estimaciones de $\alpha$ de Cronbach, fue de 0,73 , lo que sugiere un grado aceptable de coherencia entre los nueve elementos. La validez de contenido de la rúbrica de Pck fue establecido por una revisión a fondo de un panel de tres profesores. Además, como un medio para poner a prueba la validez del constructo de matriz de valoración, se llevó a cabo el análisis factorial confirmatorio para los dos componentes pre-definidos, la comprensión de los estudiantes y las estrategias de instrucción.

La segunda tendencia corresponde a estudios de casos, en donde se privilegia la comprensión del pck en relación con lo sucedido en el aula de clase y lo que acontece en los momentos de planificación, acción y en algunos el de reflexión.

Cuéllar, Rodríguez y Garritz (2015) analizan un caso de estudio de un profesor en formación, con el fin de reconocer su desarrollo del Pck; el trabajo fue de tipo cualitativo, se tuvo en cuenta las Representación del Contenido (ReCo) con respecto a los aportes realizados por Loughran, Mulhall y Berry (2004), con relación al concepto de biodiversidad. En este estudio se reconoce de manera importante la influencia que tiene el profesor en los estudiantes sobre la enseñanza y la relevancia que representa el concepto a enseñar.

En el trabajo realizado por González y Rossi (2015), de tipo cualitativo e interpretativo, se intenta conocer el PcK de cuatro docentes universitarios con respecto al concepto de mitosis, se tuvo en cuenta un cuestionario de representación del contenido (ReCo) y una observación de clases para elaborar el repertorio de experiencia profesional y pedagógica (Re-PyP), así, estos instrumentos fueron tenidos en cuenta por los aportes de Loughran et al. (2004; 2012). El instrumento (ReCo) contó con 8 preguntas que estaban dirigidas hacia componentes específicos: las preguntas 1, 2 y 3 abordaron la orientación en la enseñanza; las preguntas 4 y 5 la comprensión por parte de los estudiantes; las preguntas 6 y 7 el conocimiento de estrategias y por último la pregunta 8 acerca de la evaluación.

En el estudio de Mthethwa-Kunene, Oke Onwu, y De Villiers (2015) se utilizó un enfoque de investigación cualitativo. Se trabajó con cuatro estudios de casos, donde se analizaron tres componentes del Pck relacionado con el tema de genética. Para el componente del conocimiento de la materia por parte del maestro se tuvieron 
en cuenta entrevistas, planeación y observaciones de las clases, además de vincular mapas conceptuales. Para el componente del conocimiento pedagógico se tuvieron en cuenta cuestionarios y entrevistas. Para el componente del conocimiento de las preconcepciones y las dificultades de aprendizaje de los estudiantes, se tuvieron en cuenta las planeaciones de los profesores, entrevistas, y observaciones de las clases. También para conocer el desarrollo del Pck en cada maestro se consideraron apropiadas entrevistas, esto con el fin de reconocer este conocimiento en cada uno de los docentes. Los estudios de pruebas de contenido fueron revisados por profesores universitarios de biología. Los autores reconocen que el PCK en una construcción propia que se da en cada uno de los profesores.

La metodología empleada por Chapoo, Thathong y Halim (2014) en el estudio fue de tipo cualitativo, a través de tres profesores de biología que enseñaban en una escuela secundaria pública; para cada uno se realizó un cuestionario y posteriormente una entrevista, luego de esto se observó un video de la clase y el investigador entrevistó a estudiantes en el aula; seguidamente, se realizó otra entrevista a los docentes de tipo semi-estructurada, los análisis de estos datos se fueron: 1) análisis de cada uno de los casos, y 2) comparaciones entre ellos.

La investigación de Garnica y Roa (2012) se desarrolló dentro el paradigma interpretativo cualitativo, donde se realizó análisis de PCK a dos profesoras de biología de secundaria con respecto al concepto de fotosíntesis. Para la recolección de datos se utilizó una entrevista semi-estructurada, un cuestionario y un registro de video. Los autores tienen en cuenta varios momentos dentro de la investigación: 1) revisión bibliográfica; 2) diseño de la investigación; 3) establecer preguntas tanto para la entrevista como para el cuestionario, y 4) codificación de la información específica para cada instrumento y elementos del (Conocimiento Didáctico del Contenido sobre fotosíntesis) CDCF. En la sistematización de datos se hizo una transcripción de las respuestas brindadas en cada uno de los instrumentos empleados. Un hallazgo que se destaca es que hay diferencias en el CDCF de las profesoras y también se reconoce que algunos elementos de este conocimiento permiten construir y desarrollar otros.

Brown, Friedrichesen y Abell (2012) desarrollaron un estudio de caso con cuatro profesores de secundaria, las fuentes de datos incluyen una lección de planificación de tareas y dos ciclos de entrevistas de observación durante el año de práctica de los participantes; así, el estudio se configura como longitudinal de un programa de formación de postgrado, con profesores que tienen formación en biología y el curso se centró en el modelo 5S. En el proyecto de investigación se reconocen los planteamientos de Magnusson et al. (1999) sin embargo, se diseñaron protocolos de entrevista para obtener el conocimiento de cada uno de los componentes del PCK de futuros profesores, únicamente los datos relacionados con sus orientaciones enseñanza de las ciencias, el conocimiento de los alumnos y el conocimiento de la secuencia de instrucción.

Park y Chen (2012) incluyen observaciones de aula, entrevistas semi-estructuradas, planes de estudio, materiales didácticos y muestras de trabajo de los estudiantes. Los datos fueron analizados a través de tres enfoques diferentes: a) un análisis en profundidad del PCK explícito; b) enfoque enumerativo, y c) el método comparativo constante. Las observaciones en el aula se hicieron en las tres secciones de Biología General impartido por tres profesores que participaron en el estudio, y una clase de Biología avanzada por el cuarto docente, se trabajó con el concepto de fotosíntesis y herencia. En este estudio se realizó una adición a la metodología del examen de PCK, demostrando la posibilidad de utilizar mapas de PCK como una herramienta para hacerlo más visible, explícito y accesible. La investigación se ha apoyado en CoRe y PaPers como herramientas metodológicas útiles no solo para capturar PCK de maestros, que suele ser tácito, sino para facilitar la reflexión sobre la práctica y el desarrollo profesional (Garritz, Porro, Rembado, y Trinidad, 2005; Loughran et al., 2006; 2008).

Cohen y Yarden (2009) examinaron el conocimiento pedagógico del contenido de los profesores en relación con el tema de la célula y la formación de las relaciones macro-micro, diez años después de la publicación de la modificación del plan de estudios. Los profesores de este estudio participaron en tres grupos focales $(n=59)$ y un taller $(n=12)$. Además, seis profesores experimentados fueron entrevistados en el curso de este estudio. Las entrevistas y los debates del taller fueron grabados en audio, y las cintas fueron transcritas y analizadas. Seis herramientas diferentes se desarrollaron en el transcurso de este estudio con el fin de sondear el PCK en profesores junior-secundaria, de acuerdo con los cinco componentes sugeridos por Magnusson et al. (1999).

En la investigación de Kapyla, Heikkinen, y Asunta (2009) el objetivo fue estudiar el efecto de la cantidad y calidad del conocimiento del contenido en el conocimiento pedagógico del contenido. Se tuvo en cuenta el concepto de fotosíntesis biológica, utilizando el crecimiento de la planta como ejemplo. La muestra del estudio consistió en diez profesores estudiantes de primaria y diez de secundaria (biología); para el estudio se utilizaron cuestionarios, tareas, preparación de las clases y una entrevista para recoger datos. 
Si bien en la literatura se reporta la necesidad de pensar el PCK desde una mirada integradora de sus componentes, las investigaciones, por ejemplo, de Melanie (2013) solo retoman dos de los componentes y, además de que se ha insistido en la comprensión del Pck como un saber que se deriva de la práctica, este tipo de investigaciones privilegia más que todo el nivel declarativo que las propias construcciones de los profesores.

Respecto a los instrumentos que se utilizan en el desarrollo de las investigaciones, es importante señalar que estos se articulan a las diversas perspectivas en que se comprenden con respecto al propio Pck; en este sentido se desarrollan investigaciones más en el orden declarativo desde el pensamiento de los profesores, para lo cual se utilizan instrumentos tales como entrevistas pre y post, pruebas escritas, cuestionarios, mapas conceptuales, planificación (Cuéllar, Rodríguez y Garritz, 2015; Großschedl, Harms, Kleickmann y Glowinski, 2015; Juttner, Boone, Park y Neuhaus, 2013; Schmelzing, van Driel, Juttner, Brabdenbusch, Sandmann y Neuhaus, 2013; Park y Chen, 2012; Garnica y Roa, 2012; Kapyla, Markku, Heikkinen, Jussi-Pekka y Tuula, 2009; Cohen y Yarden 2009). Un segundo grupo de estudios ubica el PCK no solo en el nivel declarativo, sino que reconocen que en la práctica misma es posible comprender su configuración. En este grupo se suelen tener en cuenta otro tipo de instrumentos para la recolección de datos tales como observaciones de clase, grabaciones de estas, también se tienen en cuenta los instrumentos mencionados anteriormente (González y Rossi, 2015; Mthethwa, Onwu y de Villiers, 2015; Chapoo, Thathong y Halim, 2014; Brown, Friedrichesen y Abell, 2012; Park, Jang, Chen y Jung, 2011).

\section{Acerca del PCK y el desarrollo profesional}

En las investigaciones se establece la necesidad de articular los desarrollos del programa del PCK, en los procesos de formación de maestros o de actualización de maestros en ejercicio profesional. Cohen y Yarden (2009) plantean que la caracterización de este conocimiento en los docentes en relación con la enseñanza y el aprendizaje sobre el tema celular en la escuela secundaria puede servir como base para el diseño de talleres para desarrollo profesional de los profesores, así como los programas de formación de pre-servicio que pueden ayudar a cerrar la brecha entre la corriente del PCK de los profesores y las dificultades de comprensión de los estudiantes, especialmente en términos de comprensión de las relaciones micro-macro dentro de los organismos vivos.
Park, Jang, Chen y Jung (2011) plantean que teniendo en cuenta que los maestros están en el centro de la reforma en la educación científica (Bybee, 1993), se sugiere que los esfuerzos de reforma pueden centrarse de forma productiva en el desarrollo del pck de profesores. Además la excelencia en la enseñanza, debe incluir la evaluación del PCK, ya que parece que este conocimiento es fiable y que predice lo que un maestro sabe y lo que el maestro está haciendo en el aula. Si resulta que pck es un factor crítico que permite a los profesores poner en práctica la ciencia orientada hacia la reforma de la enseñanza, los programas de formación del profesorado tendrán que integrar estrategias para mejorar su desarrollo.

Park, Jang, Chen y Jung (2011) plantean que además de los temas específicos, el pck está en el contexto específico, individual e idiosincrásico hasta cierto punto, incluso para aquellos que enseñan en un contexto de enseñanza similar (Park y Oliver 2008a; Loughran et al., 2006). Con el fin de evaluar el PCK de una manera integral, deben ser considerados también esas características. Teniendo en cuenta que la rúbrica Pck es un conjunto de criterios para cada uno de suscomponentes, dicen Park et al. (2011) que la rúbrica proporcionó a los investigadores la posibilidad de tener en cuenta el contexto dado, el contenido y la experiencia de un profesor y, por lo tanto, evaluar PCK de un maestro de manera holística. Para evaluar este conocimiento usando la rúbrica, se tienen que hacer inferencias a partir de lo que un maestro dice, cree y hace. A este respecto, la calificación de los investigadores y su formación en la puntuación debe ser asegurada para establecer la credibilidad de la evaluación del PcK. Así, el uso de la matriz de valoración para evaluar este conocimiento no es perfecto, pero razonable; en consonancia con esto, varios estudiosos de la educación científica demostraron que este puede evaluarse utilizando una medida de tipo matriz de valoración (por ejemplo, Lee et al., 2007; Taylor y Gess-Newsome, 2007).

Kapyla, Heikkinen y Asunta (2012) consideran que el PcK tiene mucho potencial en la formación del profesorado, en este sentido los estudios deben ser lo suficientemente simples para guiar la práctica, ayudar a captar rápidamente la idea y dar herramientas conceptuales simples para la planificación de las clases. En la enseñanza en Jyväskylä se ha utilizado PCK como una especie de lista de verificación. Un enfoque posible es ofrecido por Loughran, Mulhall, y Berry (2004) y Loughran et al. (2001) con su tabla de representación de contenidos combinada con la experiencia profesional y pedagógica. Sin embargo, no funciona igual con el instrumento de repertorios de contenido, ya que parece ser demasiado complicado para 
la formación inicial del profesorado. Esta investigación apoya la opinión de que se debe enseñar PCK durante la formación del maestro, tanto en los estudiantes-profesores de primaria y secundaria, además de fortalecer en los profesorores en formación de primaria el trabajo sobre el CK, ya que este se constituye en un obstáculo importante en la planificación de la lección.

Hay algunos programas de formación de profesores que utilizan PCK como un concepto central en sus programas de educación. Zembal-Saul, Starr y Krajcik (1999) lo han utilizado en la unidad de enseñanza de las ciencias para profesores de primaria. Ellos utilizaron la clasificación de las áreas de conocimiento de maestros de acuerdo a Magnusson et al. (1999). Un programa similar para la formación de los profesores de ciencias de secundaria ha sido presentado por Niess y Scholtz (1999). Los resultados de estos y otros trabajos de investigación (Jones y Moreland, 2004; Mason, 1999) muestran que el uso de pск como marco ha sido eficaz en el desarrollo del pensamiento y la comprensión de lo que podría ser la enseñanza eficaz.

Brown, Friedrichesen y Abell (2012) plantean que la investigación sobre la naturaleza del desarrollo Pck tiene el potencial de informar el diseño de programas de formación docente. Los futuros profesores de secundaria ingresan a los programas de formación del profesorado con orientaciones sólidas para la enseñanza de la ciencia que se basan principalmente en sus experiencias de la propuesta denominada K-16. La perspectiva de los futuros docentes de ciencias sobre las orientaciones de enseñanza conforma significativamente la forma de como se da significado a las experiencias en cursos de metodología y experiencias de campo, mientras que las orientaciones de enseñanza de la ciencia podrían ser un gran apoyo para el aprendizaje futuro, sin que actué como una barrera para el conocimiento.

Schmelzing et al. (2013) analiza en el contexto de la formación del profesorado de biología la prueba de pск que se desarrolló en su estudio y que podría ser utilizada para la certificación de maestros, así como para la autoevaluación para el tema de la sangre y el sistema cardiovascular humano. Por otra parte, el desarrollo de los ítems de este conocimiento puede utilizarse como un método para la formación de docentes. Chapoo, Thathong y Halim (2014) señalan que el mayor desarrollo de PCK del profesor podría ser apoyado, ya que aprenden de su práctica. Se sugiere que hay una necesidad urgente por mejorar los métodos de enseñanza de los profesores de biología, mientras que se fomenta un mejor entendimiento de los propósitos fundamentales de la educación científica, el plan de estudios y el contenido de la materia.
Mthethwa-Kunene, Onwu y De Villiers (2015) señalan que las implicaciones educativas de los resultados de este estudio sugieren que las habilidades del maestro sobre el pensamiento reflexivo deben ser incluidas como un resultado de cualquier programa de formación docente para ser evaluados. Además, los programas de formación del profesorado en Suazilandia deben documentar las listas de los conceptos erróneos de los estudiantes y marcos alternativos de conceptos científicos que se consideran generalmente difícil de aprender como una forma de enriquecer el pck de los docentes de ciencia en formación y en servicio. Großschedl, Harms, Kleickmann y Glowinski (2015) plantean que las oportunidades de aprendizaje (por ejemplo, un curso de educación superior) para el PcK y Pk deberán coordinarse mejor en la formación docente, por ejemplo, mediante cursos comunes realizados por los profesores de biología, pedagogía o psicología, o trabajando en las tareas que requieren la integración del conocimiento de estas diferentes disciplinas (en Alemania, Müller, y Harms, 2009, citado en Großschedl, Harms, Kleickmann y Glowinski, 2015). Por lo tanto, la fusión de PCK y PK podría ser mejorada. Por último, encontramos en la experiencia docente una oportunidad de aprendizaje relevante especialmente para el desarrollo de PCK, que legitima a posteriori la decisión de aumentar la proporción de formación práctica en la educación superior. La futura investigación tiene que descubrir aún con más detalle las oportunidades de aprendizaje, con el fin de hacer declaraciones acerca de cómo mejorar la formación del profesorado.

Brown, Friedrichesen y Abell (2012) precisan que los hallazgos de su estudio indican que los formadores de profesores deben provocary desafiar las orientaciones de enseñanza de la ciencia de los futuros profesores. Los formadores de docentes deben hacer explícitas las perspectivas acerca de la enseñanza de la ciencia y ayudar a los futuros profesores examinar sus propios puntos de vista a la luz de la práctica de orientación reformista. Russell y Martin (2007) sugieren que la preparación de los maestros podría ser mejor visto como un proceso de cambio conceptual. Desde esta perspectiva, los cursos de métodos de ciencia y experiencias de campo deberían ayudar a los futuros profesores a crear las condiciones para conflicto cognitivo y proporcionar oportunidades para la reflexión en la opinión, y la práctica podría ayudar a los futuros maestros volver a considerar su puntos de vista.

Los estudios objeto de revisión insisten en la necesidad de incrementar el número de investigaciones acerca del PCK en profesores de biología, dada la importancia de su relación con el desarrollo profesional del profesor de biología. Cohen y Yarden (2009) han realizado numerosos 
estudios que examinan PCK de los docentes en cuanto a la relación entre niveles micro y macro en el contexto de la química (De Jong y van Driel, 2003; van Driel y Verloop, 2002), mientras que relativamente no se ha dado énfasis al estudio de este conocimiento en los docentes en el contexto de la biología en general y sus diferentes niveles de organización.

Mthethwa-Kunene, Onwu y De Villiers (2015) plantean que las similitudes y diferencias en los perfiles de pск de los cuatro profesores fueron resaltadas y los hallazgos llevaron a la conclusión que el pck es una forma compleja del conocimiento del profesor construido por ellos mismos en conveniencia con su comprensión del conocimiento del contenido específico de la materia, usando múltiples estrategias idiosincráticas para mejorar el aprendizaje de los estudiantes.

Juttner, Boone, Park y Neuhaus (2013) proponen que la investigación futura debería centrarse también en la relación entre el ск у PсK del maestro y en su práctica. En particular, la influencia de los conocimientos de los maestros sobre los estudiantes consecuente con lo asumido en la literatura, pero no se examina empíricamente lo suficiente para sacar un resultado concluyente (Baumert et al., 2010; Gess-Newsome, 1999; Hill et al., 2005). A pesar de las diferentes conceptualizaciones de Pck (Abell, 2007; Park et al., 2011; Baumert et al., 2010; Baxter y Lederman 1999; Berry et al., 2008; Blomeke et al., 2008; Hashweh, 2005; Hill et al., 2005; Magnusson et al., 1999), existe un acuerdo con respecto a dos aspectos críticos del Pck: conocimiento de la comprensión de los estudiantes y el conocimiento de las estrategias de enseñanza (Park y Oliver, 2008; Baumert et al., 2010; Lee y Luft, 2008; van Driel et al., 1998).

Mthethwa-Kunene, Onwu y De Villiers (2015) señalan que algunas de las limitaciones de este estudio incluyen la falta de evidencia sobre la eficacia del PсK individual de los maestros en el aprendizaje y rendimiento de los estudiantes acerca de la genética.

\section{Conclusiones}

Respecto a la conceptualización del Pck, en los artículos objeto de esta revisión se evidencia la vigencia de los planteamientos de Shulman (1987) en relación con la configuración de este conocimiento como singular del profesor; sin embargo, en los artículos no se observa un avance en la propia reconstrucción del pck en consideración a las condiciones actuales del ser profesor o en relación con las propias limitaciones que plantea Shulman.

En las investigaciones revisadas a través de la lectura de los artículos es posible señalar que para comprender el PCK, los investigadores utilizan dos modelos: el propuesto por Magnusson et al. (1999) o el planteado por Park y Oliver (2007). Los trabajos ubicados en el primer grupo (Cuellar, Rodríguez y Garritz, 2015; González y Rossi, 2015; Mthethwa-Kunene, Onwu y de Villiers (2015); Chapoo, Thathong y Halim, 2014; Schmelzing, van Driel, Juttner, Brabdenbusch, Sandmann, y Neuhaus, 2013; Brown, Friedrichesen y Abell, 2012; Cohen y Yarden, 2009; Käpyläa, Pekka Heikkinenb y Asuntaa, 2009) establecen una relación entre los componentes, privilegiando el componente de orientaciones sobre la enseñanza como aquel que articula a los cuatro componentes restantes. $\mathrm{El}$ segundo grupo de trabajos abordan la idea del рск desde una perspectiva de integración de todos los componentes. (Juttner, Boone, Park y Neuhaus, 2013; Park, Jang, Chen y Jung, 2011; Park y Chen, 2012).

En las investigaciones reportadas a través de los artículos revisados se evidencia que el pck se configura a través del tipo y número de relaciones entre los componentes. En este sentido, esto implica que este conocimiento es más que la suma de los componentes constituyentes y que las relaciones dinámicas entre estos configuran un PCK singular y que su calidad depende de las emergencias que se suceden de las interacciones.

Respecto a los componentes del рск que se presentan con mayor regularidad en las investigaciones, corresponden a las estrategias de enseñanza y el conocimiento de los estudiantes, Cuellar, Rodríguez, y Garritz, (2015); González y Rossi (2015); Mthethwa-Kunene, Onwu y de Villiers (2015); Chapoo, Thathong y Halim (2014); Park y Chen (2012); Garnica y Roa (2012); Brown, Friedrichesen y Abell (2012); Cohen y Yarden (2009); Käpyläa, Pekka Heikkinenb y Asuntaa (2009); Park y Oliver (2008a); Park Soonhye y Chen Ying-(2012); Soonhye Park, Jeong-Yoon Jang, YingChin Chen y Jinhong Jung (2011)

Respecto a los componente que presentan menor reconocimiento por parte de los maestros en la configuración del Pck, se encuentra el componente del currículo, como en Großschedl, Harms, Kleickmann y Glowinski (2015); Chapoo, Thathong y Halim (2014); Park y Chen (2012); Garnica y Roa (2012); Cohen y Yarden (2009); Käpyläa, Pekka Heikkinenb y Asuntaa (2009); en el componente de evaluación están González y Rossi (2015); Großschedl, Harms, Kleickmann y Glowinski (2015); Chapoo, Thathong y Halim (2014); Park y Chen (2012); Garnica y Roa (2012); Cohen y Yarden (2009).

Sobre los instrumentos que se utilizan en el desarrollo de las investigaciones, es importante señalar que estos se articulan a las diversas perspectivas en que se comprende el propio PCK; en este sentido, se desarrollan 
investigaciones, más en el orden declarativo desde el pensamiento de los profesores, para lo cual se utilizan instrumentos tales como entrevistas pre y post, pruebas escritas, cuestionarios, mapas conceptuales, planificación, como en Cuéllar, Rodríguez y Garritz (2015); Großschedl, Harms, Kleickmann y Glowinski (2015); Juttner, Boone, Park y Neuhaus (2013); Schmelzing, van Driel, Juttner, Brabdenbusch, Sandmann y Neuhaus (2013); Park y Chen (2012); Garnica y Roa (2012); Kapyla, Markku, Heikkinen, Jussi-Pekka y Tuula (2009); Cohen y Yarden (2009))

Un segundo grupo de estudios ubican el Pck no solo en el nivel declarativo sino que reconocen que en la práctica misma es posible comprender su configuración; en este grupo se suelen tener en cuenta otro tipo de instrumentos para la recolección de datos tales como observaciones de clase, grabaciones, además de los instrumentos mencionados anteriormente, González y Rossi (2015); Mthethwa, Onwu y de Villiers (2015); Chapoo, Thathong y Halim (2014); Brown, Friedrichesen y Abell (2012); Park, Jang, Chen y Jung (2011).

La lectura de los artículos evidencia que el Pck en profesores de biología se constituye en un conocimiento en construcción y que se hace necesario desarrollar investigaciones que permitan relacionar los resultados obtenidos para configurar, (para el caso de los conceptos estructurante de la biología), un conocimiento profesional que aporte en cualificar las prácticas de enseñanza y de aprendizaje en este campo.

Es importante señalar que en los artículos objeto de revisión no se hace explicito un desarrollo de orden epistemológico acerca del pck asunto que merece una reflexión en tanto que la comprensión acerca de su propia naturaleza epistémica se relaciona con los desarrollos de orden metodológico para su caracterización.

Es importante continuar desarrollando investigaciones en las que se privilegien la comprensión del pck, como una interacción de los componentes, en donde se vinculen los aspectos de emoción, afecto, sentimientos y motivación; el carácter político e ideológico de la enseñanza, el papel del profesor en la construcción de ciudadanos en una sociedad democrática y el contexto sociocultural. Por esto, se reconoce el reto propuesto por Shulman (2015), asunto que no se ve explícito en las investigaciones revisadas

Se evidencia la necesidad de desarrollar investigaciones del Pck en profesores de biología desde la práctica misma, con respecto a los propios contextos de aula e institucional que contribuyen en la constitución singular de este conocimiento, además de establecer la necesidad de articular los desarrollos del programa del pck en los procesos de formación de maestros o de actualización de maestros en ejercicio profesional.

\section{Referencias}

Abell, S. (2007). Research on Science Teacher Knowledge. En S. Abell y N. Lederman (Comps.), Handbook of research on science education (pp. 1105-1149). London: Lawrence Erlbaum Associates.

Abell, S. (2008) Twenty years later. Does Pedagogical Content Knowledge remain a useful idea?. International Journal of Science Education, 30 (10), 1405-1416.

Baumert, J., Kunter, M., Blum, W., Brunner, M., Voss, T., y Jordan, A. (2010). Teachers' mathematical knowledge, cognitive activation in the classroom, and student progress. American Educational Research Journal, 47(1), 133-180.

Baxter, J. A., y Lederman, N. G. (1999). Assessment and measurement of Pedagogical Content Knowledge. En J. Gess-Newsome y N. Lederman (Eds.), Examining Pedagogical Content Knowledge: The Construct and its Implications for Science Education (pp. 147-161). Dordrecht: Kluwer.

Berry, A., Friedrichsen, P., y Loughran, J. (Eds.). (2015). Re-examining Pedagogical Content Knowledge in science education. New York: Routledge.

Berry, A., Loughran, J. y van Driel J. (2008). Revisiting the roots of Pedagogical Content Knowledge. International Journal of Science Education, 30(10), 1271-1279.

Blömeke, S., Kaiser, G., Lehmann, R., y Schmidt, W. $\mathrm{H}$. (2008). Introduction to the issue onempirical research on mathematics teachers and their education. zDM Mathematics Education, 40, 715-717.

Borko, H., y Putnam, R. T. (1996). Learning to Teach. En D. C. Berliner y R. C. Calfee (Eds.), Handbook of Educational Psychology (pp. 673-708). New York: Macmillan.

Brown, P., Friedrichsen, P., y Abell, S. (2012). The development of prospective secondary biology teachers Pck. Journal of Science Teacher Education, 24(1), 133-155.

Bybee, R. W. (1993). Leadership, responsibility, and reform in science education. Science Educator, 2(1), 1-9. 
Chapoo, S., Thathong, K., Y Halim, L. (2014). Understanding biology teacher's Pedagogical Content Knowledge for teaching "the nature of organism". Procedia-Social and Behavioral Sciences, 116, 464-471.

Charrier, M. (2005). El conocimiento didáctico del contenido sobre la fotosíntesis. Estudio de las concepciones y de la práctica de los profesores del tercer ciclo de la educación general básica argentina. Tercer Encuentro de Investigadores en Didáctica de la Biología. Universidad de Buenos Aires, Argentina.

Charrier, M. (2009). El conocimiento didáctico del contenido sobre la fotosíntesis de dos profesores de educación secundaria argentinos. Enseñanza de las ciencias: revista de investigación y experiencias didácticas. Recuperado de www.raco.cat/index. php/Ensenanza/article/download/294424/382945.

Clermont, C. P., Krajcik, J. S., y Borko, H. (1993). The Influence of an intensive in囚service workshop on Pedagogical Content Knowledge growth among novice chemical demonstrators. Journal of Research in Science Teaching, 30(1), 21-43.

Cochran, K. F., King, R. A., y DeRuiter, J. A. (1991). Pedagogical Content Knowledge: A tentative model for teacher preparation. East Lansing, Michigan: National Center for Research on Teacher Learning.

Cohen, R. y Yarden, A. (2009). Experienced junior-high-school teachers' Pck in light of a curriculum change: "the cell is to be studied longitudinally". Science Education, 39, 131-155.

Cuellar, Z. L., Rodríguez, L., y Garritz, A. (2015). Las grandes ideas sobre biodiversidad y la ReCo de un estudiante-profesor. Visita de una profesora de la Universidad Surcolombiana. Educación química, 26(1), 2-8.

De Jong, O., y van Driel, J. (2003). Exploring the development of student teachers' PсK of the multiple meanings of chemistry topics. International Journal of Science and Mathematics Education, 2(4), 477-491.

Fischer, H. E., Borowski, A., y Tepner, O. (2012). Professional Knowledge of Science Teachers. En Second international handbook of science education (pp. 435-448). Recuperado de https://www.researchgate.net/publication/259177525_Professional_ Knowledge_of_Science_Teachers

Friedrichsen, P., Driel, J. H. V., y Abell, S. K. (2011). Taking a closer look at science teaching orientations. Science Education, 95(2), 358-376.
Garnica, S. E., y Roa, A. R. (2012). Conocimiento didáctico del contenido sobre fotosíntesis de dos profesores de los grados sexto y noveno de educación básica secundaria de un colegio privado en Bogotá-Colombia. Bio-grafía: escritos sobre la biología y su enseñanza, 5(8), 50-76.

Garritz, A., Porro, S., Rembado, F. M., y Trinidad, R. (2005). Latin-american teachers' Pedagogical Content Knowledge of the particulate nature of matter. Journal of Science Education, 8(2), 79.

Gess-Newsome, J. (1999). Secundary teachers' knowledge and beliefs about subjet matter and their impact on instruction. En J. Gess-Newsome y N. Lederman (Eds.), Examining Pedagogical Content Knowledge. The Construct and its Implications for Science Education (pp. 51-94). Netherlands: Kluwer Academic Publishers.

Gess-Newsome, J. (1999a). Pedagogical Content Knowledge: An introduction and orientation. En J. Gess-Newsome y N. Lederman (Eds.), Examining Pedagogical Content Knowledge (pp. 3-17). Netherlands: Kluwer Academic Publishers.

González, N. V., y Rossi, A. M. (2015). Conocimiento pedagógico del contenido para la enseñanza del tema mitosis: un estudio de casos con docentes universitarios de Argentina. Didáctica de las Ciencias Experimentales y Sociales, (29), 215-232.

Grossman, P. (1990). The Making of a Teacher. Teacher Knowledge and Teacher Education. Nueva York: Columbia University.

Großschedl, J., Harms, U., Kleickmann, T., y Glowinski, I. (2015). Preservice biology teachers' professional knowledge: Structure and learning opportunities. Journal of Science Teacher Education, 26(3), 291-318.

Hashweh, M. Z. (2005). Teacher pedagogical constructions: A reconfiguration of Pedagogical Content Knowledge. Teachers and Teaching: Theory and Practice, 11(3), 273-292.

Hill, H. C., Rowan, B., y Ball, D. (2005). Effects of teachers' mathematical knowledge for teaching on student achievement. American Educational Research Journal, 42(2), 371-406.

Jones, A., y Moreland, J. (2004). Enhancing practicing primary school teachers' Pedagogical Content Knowledge in technology. International Journal of Technology and Design Education, 14(2), 121-140. 
Juttner, M., Boone, M., Park, S., y Neuhaus, B. (2013). Development and use of a test instrument to measure biology teachers' Content Knowledge (ck) and Pedagogical Content Knoledge (РСK). Educational Assessment, Evaluation and Accountability, 25, 45-67.

Käpylä, M., Heikkinen, J. P., y Asunta, T. (2009). Influence of Content Knowledge on Pedagogical Content Knowledge: The case of teaching photosynthesis and plant growth. International Journal of Science Education, 31(10), 1395-1415.

Kind, V. (2015) On the beauty of knowing then not knowing. En A. Berry, P. Friedrichsen y J. Loughran (Eds.), Re-Examining Pedagogical Content Knowledge in Science Education. New York: Routledge.

Latorre, A., Rincón, D. y Arnal, J. (2003). Bases metodológicas de la investigación educativa. Barcelona: Ediciones Experiencia.

Lee, E., y Luft, J. A. (2008). Experienced secondary science teachers' representation of Pedagogical Content Knowledge. International Journal of Science Education, 30(10), 1343-1363.

Lee, E., Brown, M. N., Luft, J. A., y Roehrig, G. H. (2007). Assessing beginning secondary science teachers' PCK: pilot year results. School Science and Mathematics, 107(2), 52-60.

Loughran, J. (2012). What expert teachers do: enhancing professional knowledge for classroom practice. New York: Routledge.

Loughran, J., Mulhall P., y Berry A. (2008). Exploring Pedagogical Content Knowledge in science teacher education. International Journal of Science Education, 30, 1301-1320.

Loughran, J., Berry, A., y Mulhall, P. (2006).Understanding and Developing Science Teachers' Pedagogical Content Knowledge. Rotterdam: Sense Publishers.

Loughran, J., Milroy, P., Berry, A., Gunstone, R., y Mulhall, P. (2001). Documenting science teachers' Pedagogical Content Knowledge through papers. Research in Science Education, 31, 289-307.

Loughran, J.; Mulhall, P. y Berry, A. (2004). In search of Pedagogical Content Knowledge in science: developing ways of articulating and documenting professional practice. Journal of Research in Science Teaching, 41(4), 370-391.

Magnusson, S., Krajcik, J., y Borko, H. (1999). Nature, sources and development of Pedagogical Content Knowledge. En J. Gess-Newsome y N. G. Lederman
(Eds.), Examining Pedagogical Content Knowledge (pp. 95-132). Dordrecht: Kluwer Academic Publishers.

Mason, C. (1999) The triad approach: A consensus for science teaching and learning. En J. Gess-Newsome y N. Lederman (Eds.), Examining Pedagogical Content Knowledge. The Construct and its Implications for Science Education (pp. 277-292). London: Kluwer Academic Publishers.

Mthethwa-Kunene, E., Onwu, G. O., y de Villiers, R. (2015). Exploring biology teachers' Pedagogical Content Knowledge in the teaching of genetics in swaziland science classrooms. International Journal of Science Education, 37(7), 1140-1165.

National Research Council. (1996). National Science Education Standards. Washington: National Academy Press.

Niess, M. L. y Scholz, J. (1999). Incorporating subject matter specific teaching strategies into secondary science teacher. En J. Gess-Newsome y N. Lederman (Eds.), Examining Pedagogical Content Knowledge. The Construct and its Implications for Science Education (pp. 257-276). London: Kluwer Academic Publishers.

Park, S., y Chen, Y. C. (2012). Mapping out the integration of the components of Pedagogical Content Knowledge (PсK): Examples from high school biology classrooms. Journal of Research in Science Teaching, 49(7), 922-941.

Park, S., y Oliver, J. S. (2008). Revisiting the conceptualisation of Pedagogical Content Knowledge (PCK): PCK as a conceptual tool to understand teachers as professionals. Research in Science Education, 38(3), 261-284

Park, S., y Oliver, J. S. (2008a). National Board Certification (NBC) as a catalyst for teachers' learning about teaching: the effects of the NBC process on candidate teachers' PCK development. Journal of Research in Science Teaching, 45(7), 812-834.

Park, S., Jang, J.-Y., Chen, Y.-C., y Jung, J. (2011). Is Pedagogical Content Knowledge (PCK) necessary for reformed science teaching? Evidence from an empirical study. Research in Science Education, 41(2), 245-260.

Russell, T. y Martin, A. (2007). Learning to teach science. En S. Abell y N. Lederman (Eds.), Handbook of Research on Science Education. (pp. 1151-1176). Mahwah: Laurence Erlbaum Associates. 
Sawada, D., Piburn, M. D., Judson, E., Turley, J., Falconer, K., Benford, R., y Bloom, I. (2002). Measuring reform practices in science and mmathematics classrooms: the reformed teaching observation protocol. School Science and Mathematics, 102(6), 245-253.

Schmelzing, S., van Driel, J. H., Jüttner, M., Brandenbusch, S., Sandmann, A., y Neuhaus, B. J. (2013). Development, evaluation, and validation of a paper-and-pencil test for measuring two components of biology teachers' Pedagogical Content Knowledge concerning the "cardiovascular system". International Journal of Science and Mathematics Education, 11(6), 1369-1390.

Shulman, L. S. (1986). Those who understand: knowledge growth in teaching. Educational Researcher, 15(2), 4-14.

Shulman, L. S. (1987). Knowledge and teaching: foundations of the new reform. Harvard Educational Review, 57(1), 1-22.

Tamir, P. (1988). Subject matter and related pedagogical knowledge in teacher education. Teaching and Teacher Education, 4, 99-110.

Taylor, J., y Gess-Newsome, J. (2007). Exploring tools and methods for measuring Pedagogical Content Knowledge. En Annual Conference of the Association for Science Teacher Education, Clearwater, FL.
Uribe, J. (2011). La investigación documental y el estado del arte como estrategias de investigación en ciencias sociales. En P. Páramo (Comp.), La investigación en ciencias sociales: estrategias de investigación. Bogotá. Universidad Piloto de Colombia.

Valbuena, E. (2007). El Conocimiento didáctico del contenido biológico. Estudio de las concepciones disciplinares y didácticas de futuros docentes de la Universidad Pedagógica Nacional (Colombia). Madrid: Universidad Complutense.

van Driel, J. H., y Verloop, N. (2002). Experienced teachers' knowledge of teaching and learning of models and modelling in science education. International Journal of Science Education, 24 (12), 1255-1272.

van Driel, J. H., Verloop, N., y de Vos, W. (1998). Developing science teachers' Pedagogical Content Knowledge. Journal of research in Science Teaching, 35 (6), 673-695.

Zembal-Saul, C., Starr, M. L., y Krajcik, J. S. (1999). Constructing a framework for elementary science teaching using Pedagogical Content Knowledge. En J. Gess-Newsome y N. Lederman (Eds.), Examining Pedagogical Content Knowledge. The Construct and its Implications for Science Education (pp. 237-256). Londres: Kluwer Academic Publishers. 